\title{
Characterization of circadian blood pressure patterns using non-linear mixed effects modeling
}

\author{
Dongwoo Chae ${ }^{1,2}$, Yukyung Kim ${ }^{3}$ and Kyungsoo Park ${ }^{1 \star}$ \\ ${ }^{1}$ Department of Pharmacology, Yonsei University College of Medicine, Seoul 03722, Korea \\ ${ }^{2}$ Brain Korea 21 Plus Project for Medical Science, Yonsei University, Seoul 03722, Korea \\ ${ }^{3}$ Ministry of Food and Drug Safety, Cheongju 28159, Korea \\ ${ }^{\star}$ Correspondence: K. S. Park; Tel: +82-2-2228-1735 , Fax: +82-2-313-1894 , E-mail: kspark@yuhs.ac
}

\section{Check for updates}

Received 7 Feb 2019

Revised 5 Mar 2019

Accepted 5 Mar 2019

\section{Keywords}

Circadian blood pressure fluctuation,

Nonlinear mixed effects model, Non-dipper,

NONMEM

pISSN: 2289-0882

eISSN: 2383-5427
Characterizing the time course of baseline or pre-drug blood pressure is important in acquiring unbiased estimates of antihypertensive drug effect. In this study, we recruited 23 healthy male volunteers and measured systolic (SBP) and diastolic blood pressure (DBP) over 24 hours on an hourly basis. Using a non-linear mixed effects model, circadian rhythm observed in blood pressure measurements was described by incorporating two cosine functions with periods 24 and 12 hours. A mixture model was applied to identify subgroups exhibiting qualitatively different circadian rhythms. Our results suggested that $78 \%$ of the study population, defined as 'dippers', demonstrated a typical circadian profile with a morning rise and a nocturnal dip. The remaining $22 \%$ of the subjects defined as 'non-dippers', however, were not adequately described using the typical profile and demonstrated an elevation of blood pressure during night-time. Covariate search identified weight as being positively correlated with mesor of SBP. Visual predictive checks using 1,000 simulated datasets were performed for model validation. Observations were in agreement with predicted values in 'dippers', but deviated slightly in 'non-dippers'. Our work is expected to serve as a useful reference in assessing systematic intra-day blood pressure fluctuations and antihypertensive effects as well as assessing drug safety of incrementally modified drugs.

\section{Introduction}

Blood pressure typically exhibits a circadian rhythm with a rise in the morning and a fall in the night.[1,2] This is not true for all individuals, however, and atypical patterns associated with an absence of nocturnal decline and even a paradoxical increase have been reported. Such individuals are defined as 'non-dippers' in the literature and are known to be associated with increased risk of cerebrovascular events.[3-6] Unlike other physiological rhythms, blood pressure is known to depend on activity and sleep rather than clock time per se,[7] and 'nondipper' phenotype might arise from differences in daily activities rather than from an inherent biological polymorphism.

Copyright (C) 2019 Translational and Clinical Pharmacology

(a) It is identical to the Creative Commons Attribution Non-Commercial License (http://creativecommons.org/licenses/by-nc/3.0/).

(®) This paper meets the requirement of KS X ISO 9706, ISO 9706-1994 and ANSI/NISO Z.39.48-1992 (Permanence of Paper).

Reviewer

This article reviewed by peer experts who are not TCP editors.
From the viewpoint of clinical pharmacology, the acquisition of an unbiased estimate of drug effect in antihypertensive agents requires a set of reference values of untreated blood pressure. Such information can be acquired from randomized clinical trials, either through the control group in parallel design studies or run-in periods in crossover studies. Tanigawara et al, [8] as part of a PKPD modeling work of olmesartan and azelnidipine, built a model of circadian variation of blood pressure based on ambulatory blood pressure monitoring data acquired during the run-in period and subsequently built a drug effect model using data from the treatment period.

In addition to unbiased estimation of the drug effect of antihypertensive drugs, placebo effect on blood pressure is required for the assessment of drug safety on vital signs and other cardiovascular variables acquired during drug development. When analyses must be conducted for drug effect on blood pressure in an ad hoc manner using retrospective data originally collected for alternative purposes (such as safety monitoring), identifying different sources of blood pressure variability is difficult without 
the information on the placebo effect.

Authors of this work have previously published a report whereby physiologic relationships among mean arterial pressure (MAP), pulse pressure (PP), and heart rate (HR) were exploited to simultaneously estimate circadian variation, homeostatic feedback mechanism, and drug effect,[9] but the estimates related to circadian variation showed significant deviation from that known in the literature. This is an expected result considering the sparse sampling scheme consisting of only 5 measurements per patient over a 24 hour period, but better results would have been possible if prior information with which to perform Bayesian analyses were available.

In this context, an investigator-initiated clinical trial was undertaken in this work where blood pressure was measured on an hourly basis for 24 hours without drug treatment. The purpose of the trial was to generate data for modeling the placebo effect that predicts baseline or pre-treatment blood pressure fluctuations or circadian variation in the Korean population. In particular, we attempted to model circadian variation of not only the typical 'dippers' but also 'non-dippers'. To the authors' knowledge, numerous models have been proposed to predict the typical 'morning rise, night fall' circadian pattern but none yet to predict the atypical profile of the 'non-dippers'. We intended to fill this void by constructing a model that encompasses both subpopulations, offering a more comprehensive reference of circadian blood pressure fluctuations.

\section{Methods}

\section{Study protocol}

Out of 117 male subjects who previously participated in a bioequivalence study of a newly developed fixed-dose combination tablet of telmisartan $80 \mathrm{mg} /$ rosuvastatin $20 \mathrm{mg}, 23$ were recruited for an additional investigator-initiated clinical trial. The trial was conducted between July and September 2013 in the clinical trial center of Severance Hospital, Seoul Korea. Subjects exhibiting systolic blood pressure (SBP) greater than or equal to 150 $\mathrm{mmHg}$ or less than or equal to $100 \mathrm{mmHg}$, or diastolic blood pressure (DBP) greater than or equal to $100 \mathrm{mmHg}$ or less than or equal to $65 \mathrm{mmHg}$ were excluded from this study.

Study participants were refrained from taking excessive alcohol (> $30 \mathrm{~g} /$ day), smoking (> 10 cigars/day), and any kind of medication beginning from 7 days prior to trial initiation. Subjects were admitted to the clinical trial center 1 day before intensive blood pressure (BP) measurements and were put to bed at $11 \mathrm{PM}$. No food intake was allowed after $10 \mathrm{PM}$.

On day 1, BP measurements were taken on an hourly basis beginning at $8 \mathrm{AM}$ for 24 hours. Lunch and dinner were provided at $12 \mathrm{AM}$ and $6 \mathrm{PM}$, respectively. $\mathrm{BP}$ measurements were continued into sleep-time. On day 2, study participants were discharged after the last blood pressure measurement at $8 \mathrm{AM}$. A follow-up BP sampling was done on day 3 at 8 AM.

All BP measurements during wake hours were taken at heart
Table 1. Demographics of the study participants $(n=23)$

\begin{tabular}{cc} 
Characteristics & Values \\
Age [yrs] (median, range) & $24(22-45)$ \\
SBP $[\mathrm{mmHg}]$ (mean $\pm \mathrm{sd})$ & $119.0 \pm 13.8$ \\
DBP $[\mathrm{mmHg}]$ (mean $\pm \mathrm{sd})$ & $75.0 \pm 9.5$ \\
Weight $[\mathrm{kg}]$ (mean $\pm \mathrm{sd})$ & $67.4 \pm 8.2$ \\
Height $[\mathrm{cm}]$ (mean $\pm \mathrm{sd})$ & $171.9 \pm 6.6$ \\
\hline
\end{tabular}

SBP, Systolic blood pressure; DBP, Diastolic blood pressure.

level in a sitting position after at least 5 minutes of rest. During sleep, BP measurements were taken in a sleeping position so as to minimize unnecessary stimulations. Both SBP and DBP were collected from each measurement.

The demographics of the study participants are summarized in Table 1 .

\section{Modeling circadian rhythm}

Circadian fluctuations in SBP and DBP were modeled simultaneously using two cosine functions with periods 24 and $12 \mathrm{~h}$ as described in (1) and (2).

$$
\begin{gathered}
\operatorname{CIRC}(\mathrm{t})=\operatorname{Amp}_{24} \cdot \cos \left(\frac{2 \pi}{24}\left(\mathrm{t}-\text { Phase }_{24}\right)\right)+ \\
\operatorname{Amp}_{12} \cdot \cos \left(\frac{2 \pi}{12}\left(\mathrm{t}-\text { Phase }_{12}\right)\right) \\
\mathrm{BP}_{\text {pre }}(\mathrm{t})=\text { Mesor } \cdot(1+\operatorname{CIRC}(\mathrm{t}))+\varepsilon
\end{gathered}
$$

$\mathrm{CIRC}(\mathrm{t})$ is the circadian rhythm at $\mathrm{t}$, with reference time (i.e. $\mathrm{t}$ =0) set to $12 \mathrm{AM}$, Amp $_{24}$ Phase $_{24}$ and Amp ${ }_{12}$, Phase $_{12}$ are amplitude and phase of oscillator for periods 24 and $12 \mathrm{~h}$, respectively, assumed to differ between SBP and DBP, Mesor is the mean $\mathrm{BP}$ over $24 \mathrm{~h}$, and $\varepsilon$ is the measurement error. A mixture model was used to identify subgroups of subjects exhibiting different circadian profiles assuming a common mesor and different amplitudes and phases between subgroups. NONMEM software implements a mixture model by estimating $p_{i k}(\theta)$, the mixture probability of the kth mixture $(k=1, . ., r)$ for the $i$ th subject, and then calculating $L_{i}$, the marginal likelihood for the $i$ th subject, as $L_{i}=\sum_{\mathrm{k}=1}^{\mathrm{r}} p_{i k}(\theta) \cdot \int l_{i k}(\eta, \theta) \cdot h(\eta, \Omega) d \eta$, where $l_{i k}(\eta, \theta)$ represents the conditional likelihood given $\eta$ and $h(\eta, \Omega)$ the density function of $\eta$.

Model parameters were assumed to differ randomly among study individuals.

$$
\text { Param }=t v \text { Param } \cdot \exp (\eta)
$$

In the above, $\eta$ is defined as a random variable following a normal distribution centered at zero with an associated variance $\omega^{2}$. Param is an individual-specific parameter whereas $t v$ Param is the expected (typical) model parameter estimate.

Model building was initially done without assuming a mixture 
distribution of model parameters. This base model was defined as 'Model 1'. Next, a mixture distribution of the set of circadian parameters was assumed in 'Model 2'. The improvement in model fit with this added assumption was tested based on the comparison of objective function values.

Exploratory data analysis was undertaken whereby patient covariates of age and weight were plotted against the empirical Bayesian estimates of the model parameters to select covariate candidates showing any correlation with parameters. Covariate model building was then carried out on the selected candidates using a stepwise selection algorithm performed at the significance level of $\mathrm{p}<0.01$ for forward addition and $\mathrm{p}<0.001$ for backward deletion. The covariate-incorporated model was defined as 'Model 3'.

Additive, proportional, and combined residual error models were tested and the one offering the lowest objective function value was selected.

\section{Model validation}

Model fit was assessed using basic goodness of fit plots (DV vs. PRED, CWRES vs. PRED, CWRES vs. TIME, etc.) during the model building stage. Model comparison was done based on objective function value (OFV) and estimated standard errors of model parameters. A decline of OFV beyond the threshold value of a chi-square statistic associated with $p$-value $<0.05$ was used as evidence of model improvement. The precision of model parameter estimates was judged based on the standard errors (SE) calculated using the default covariance matrix provided by NONMEM (i.e. $R^{-1} S R^{-1}$, where $\mathrm{S}$ and $\mathrm{R}$ denote cross-product gradient and Hessian matrices, respectively). The final model was validated using a visual predictive check (VPC) procedure given 1,000 simulated datasets. Percentiles of $2.5 \%, 50 \%$, and $97.5 \%$ of observations were compared with the prediction interval of corresponding percentiles to assess whether most of the observations fell within each interval.

\section{Software}

All model parameters were estimated using NONMEM software version 7.3 and $\mathrm{R}$ and RStudio were used for data exploration and visualization.

\section{RESULTS}

OFV difference between Model 1 and 2 was highly significant $(\Delta \mathrm{OFV}=37.1, \mathrm{p}<0.0001)$. Weight was incorporated into Mesor for SBP as follows, leading to an OFV decline of 18.9 ( $\mathrm{p}<$ $0.0001)$ :

$$
\text { tvMesor } r_{S B P}=\text { Mesor }_{S B P} \cdot \exp (\theta \cdot(W T-70))
$$

Where tvMesor $r_{S B P}$ represents the typical estimated mesor for SBP, $M e s o r_{S B P}$ the value of tvMesor ${ }_{S B P}$ evaluated at the median weight (WT) of $70 \mathrm{~kg}, \theta$ the covariate coefficient, and $\eta$ the unexplained deviation from the population mean. Table 2 shows the parameter estimates acquired from fitting Model 3 to data. In the typical subject group (i.e. 'dippers') comprising $78 \%$ of the study subjects, Phase $_{24}$ and Phase $_{12}$ for SBP were 14.6 and 9.81 hours, and those for DBP were 14.3 and 9.18 hours, indicating SBP and DBP were in phase as expected. Our results were in close agreement with values reported in the literature [8] (with estimates of Phase ${ }_{24}$ and Phase ${ }_{12}$ for SBP reported as 14.32 and 8.93 hours). The acrophases of the minor subject group (i.e. 'non-dippers') showed no resemblance to those of the typical subject group, however, and returned estimates of 24.7 and 12.8 hours for SBP and 27.3 and 12.0 hours for DBP for Phase $_{24}$ and Phase $_{12}$, respectively.

Random interindividual variability was incorporated into Mesor in both subject groups and $A m p_{24}$ in the typical subject group. Incorporation of random interindividual errors into other model parameters or into those of the 'non-dippers' did not lead to OFV decline associated with $\mathrm{p}<0.05$.

Table 2. Estimation result of the final model (Model 3)

\begin{tabular}{|c|c|c|c|}
\hline Parameter & \multicolumn{3}{|c|}{ Population estimate (\%RSE) } \\
\hline \multicolumn{4}{|c|}{ Structural Parameters } \\
\hline & & Dippers & Non-dippers \\
\hline$A m p_{24}(\%)$ & $\begin{array}{l}\text { SBP } \\
\text { DBP }\end{array}$ & $\begin{array}{l}2.94(23 \%) \\
2.14(33 \%)\end{array}$ & $\begin{array}{l}2.72(29 \%) \\
7.31(20 \%)\end{array}$ \\
\hline Phase $_{24}$ (h) & $\begin{array}{l}\text { SBP } \\
\text { DBP }\end{array}$ & $\begin{array}{l}14.6(4 \%) \\
14.3(4 \%)\end{array}$ & $\begin{array}{l}24.7(9 \%) \\
27.3(1 \%)\end{array}$ \\
\hline$A m p_{12}(\%)$ & $\begin{array}{l}\text { SBP } \\
\text { DBP }\end{array}$ & $\begin{array}{l}2.98(14 \%) \\
5.93(7 \%)\end{array}$ & $\begin{array}{l}2.05(31 \%) \\
1.48(46 \%)\end{array}$ \\
\hline Phase $_{12}$ (h) & $\begin{array}{l}\text { SBP } \\
\text { DBP }\end{array}$ & $\begin{array}{l}9.81(3 \%) \\
9.18(3 \%)\end{array}$ & $\begin{array}{l}12.8(4 \%) \\
12.0(10 \%)\end{array}$ \\
\hline Mesor $(\mathrm{mmHg})$ & $\begin{array}{l}\text { SBP } \\
\text { DBP }\end{array}$ & \multicolumn{2}{|c|}{$\begin{array}{r}119(1 \%) \\
69.5(2 \%)\end{array}$} \\
\hline$\theta$ & \multicolumn{3}{|c|}{$0.0067(22 \%)$} \\
\hline $\begin{array}{l}\text { Proportion of major } \\
\text { subject group }\end{array}$ & \multicolumn{3}{|c|}{$78.3(11 \%)$} \\
\hline \multicolumn{4}{|c|}{ Variance Parameters } \\
\hline$\omega_{\text {Mesor }}^{2}(\mathrm{CV} \%)$ & $\begin{array}{l}\text { SBP } \\
\text { DBP } \\
\text { Correl }\end{array}$ & & $\begin{array}{c}5.6(16 \%) \\
9.6(12 \%) \\
0.47\end{array}$ \\
\hline$\omega_{A M P_{24}}^{2}(\mathrm{CV} \%)$ & $\begin{array}{l}\text { SBP } \\
\text { DBP }\end{array}$ & $\begin{array}{r}70.3(21 \%) \\
103.7(26 \%)\end{array}$ & $\begin{array}{l}0 \text { FIX } \\
0 \text { FIX }\end{array}$ \\
\hline \multicolumn{4}{|c|}{ Residual Variability } \\
\hline$\sigma_{P r o}^{2}(\mathrm{CV} \%)$ & \multicolumn{3}{|c|}{$0.26(14 \%)$} \\
\hline$\sigma_{\text {Add }}^{2}(\mathrm{mmHg})$ & \multicolumn{3}{|c|}{$14.1(24 \%)$} \\
\hline
\end{tabular}

Amp $_{24}$, Phase $_{24}$ and $A m p_{12}$, Phase $_{12}$ : amplitude and phase of oscillator for periods 24 and $12 \mathrm{~h}$, respectively.

Mesor: the mean BP over $24 \mathrm{~h}$.

$\theta$ : the covariate coefficient, defined as below:

$$
\text { tvMesor }_{S B P}=\text { Mesor }_{S B P} \cdot \exp (\theta \cdot(W T-70)) ; \text { Mesor }_{S B P}=119
$$

tvMesor ${ }_{S B P}$ : the typical estimated mesor for SBP.

Mesor $_{S B P}$ : the value of tvMesor $r_{S B P}$ evaluated at the median weight (WT) of $70 \mathrm{~kg}$. $\sigma_{P r o}^{2}=$ variance of proportional residual error; $\sigma_{A d d}^{2}=$ variance of additive residual error.

SBP, Systolic blood pressure; DBP, Diastolic blood pressure. 
The combined error model incorporating both proportional and additive error components seemed to best describe our data.

The typical predicted values of SBP and DBP based on the final model (i.e., model 3 ) of each subject group are graphically depicted in Figure 1. There seems to be a tendency of lower me- sor in the 'non-dipper' subject group, but a post-hoc comparison of mesor between 'dippers' and 'non-dippers' failed to show a statistically significant difference when evaluated based on two-sample t-tests, with p-values of 0.078 and 0.079 for SBP and DBP, respectively.

Figure 2 shows predictions superimposed on observations,

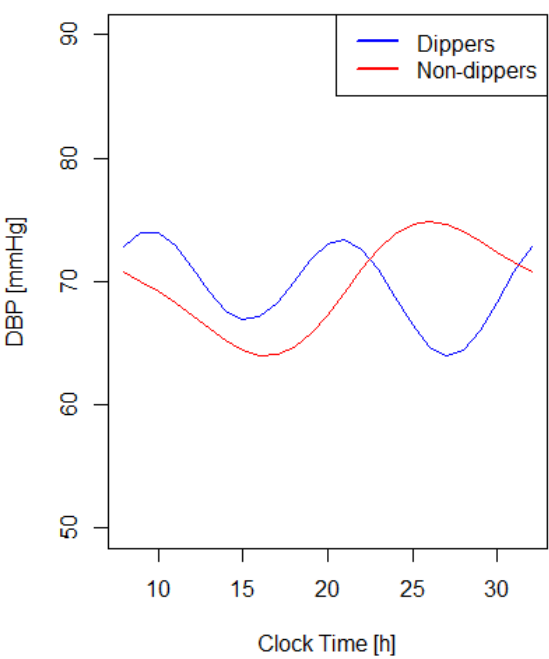

Figure 1. Typical circadian profiles of 'dippers' (blue) and 'non-dippers' (red) subject groups in SBP (left) and DBP (right) (SBP, Systolic blood pressure; DBP, Diastolic blood pressure).

Dippers
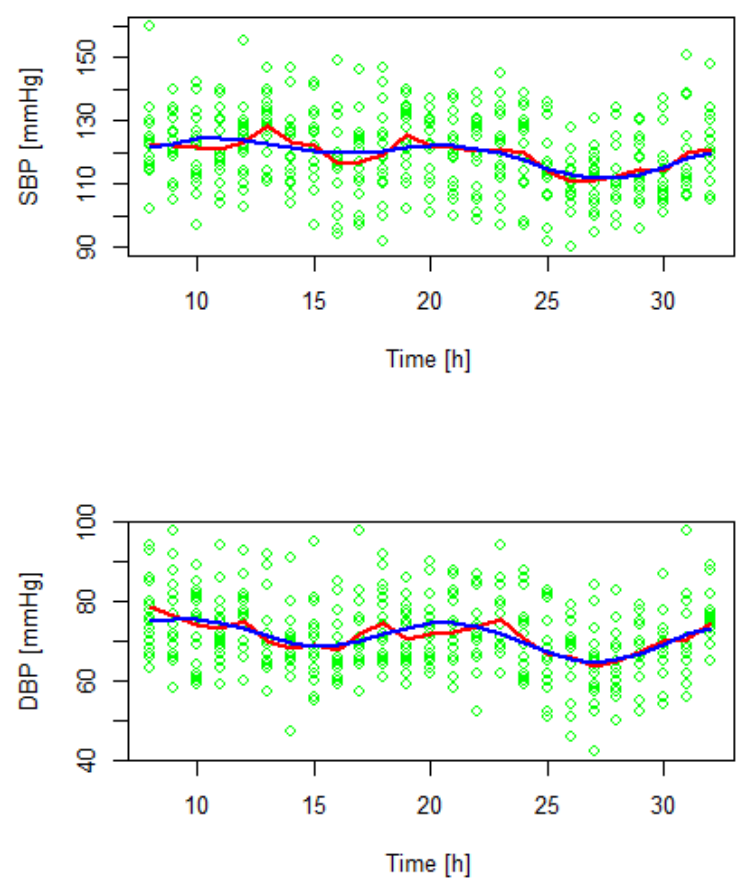

Non-Dippers
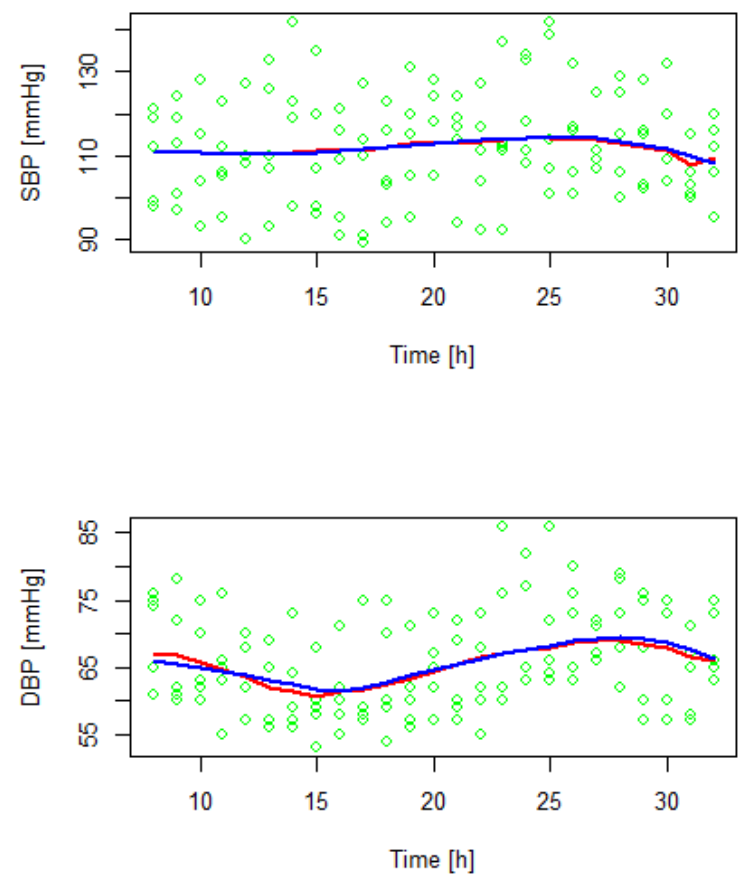

Figure 2. Goodness of fit plots showing smoothed predictions (blue line), superimposed on smoothed observations (red line) and original observations (green dots). (SBP, Systolic blood pressure; DBP, Diastolic blood pressure). 
Dippers

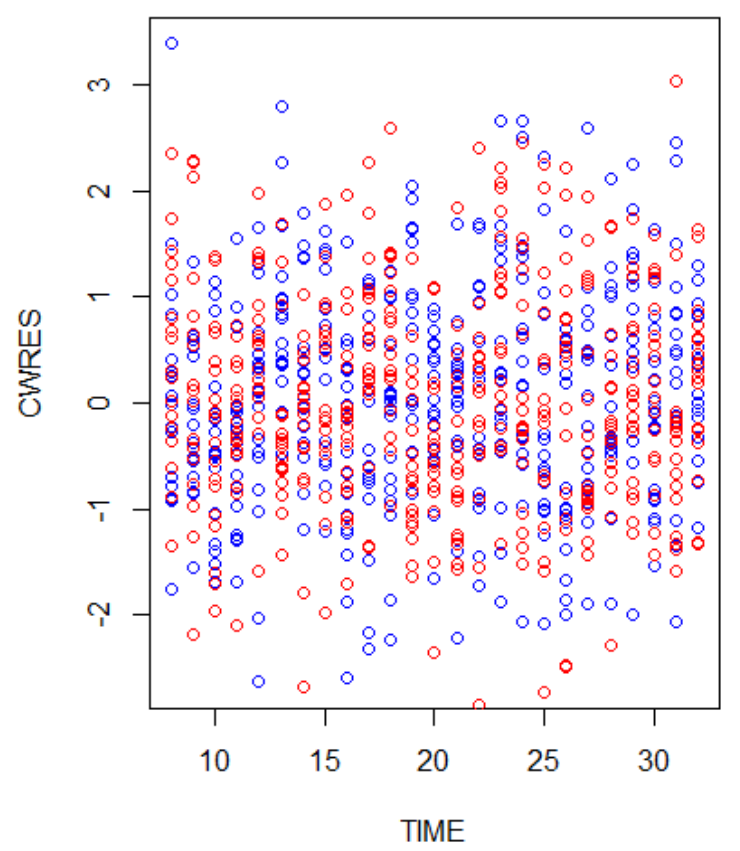

Dippers (SBP)

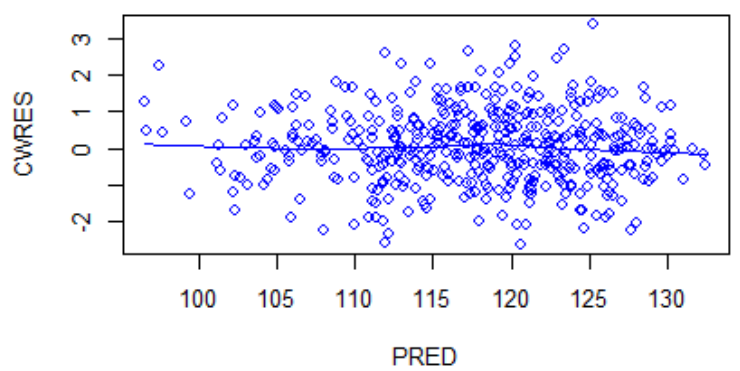

Dippers (DBP)

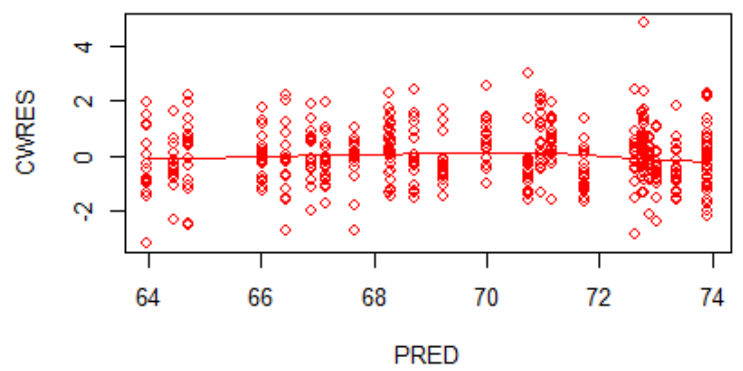

Non-Dippers

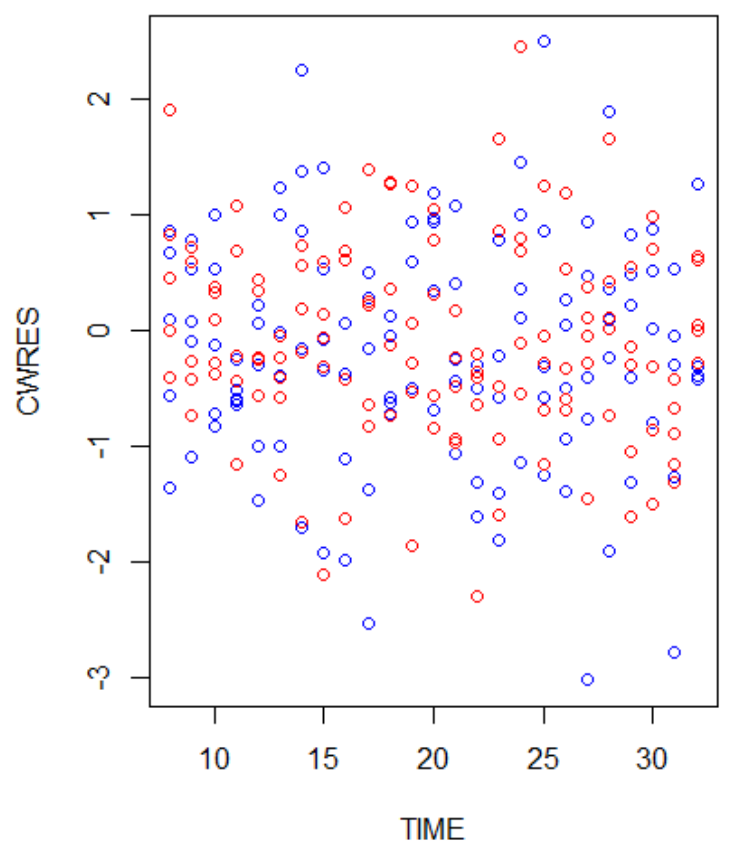

Non-Dippers (SBP)

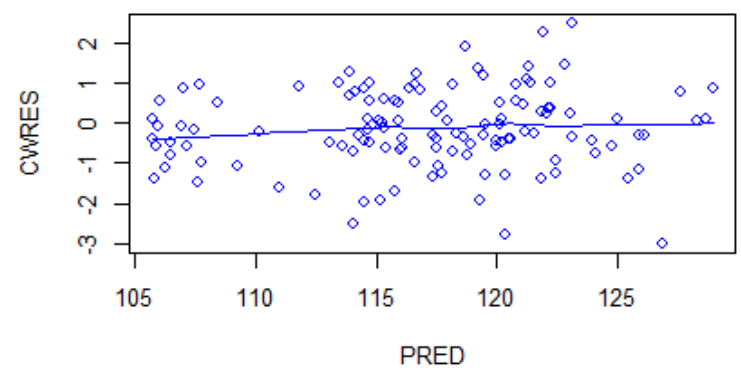

Non-Dippers (DBP)

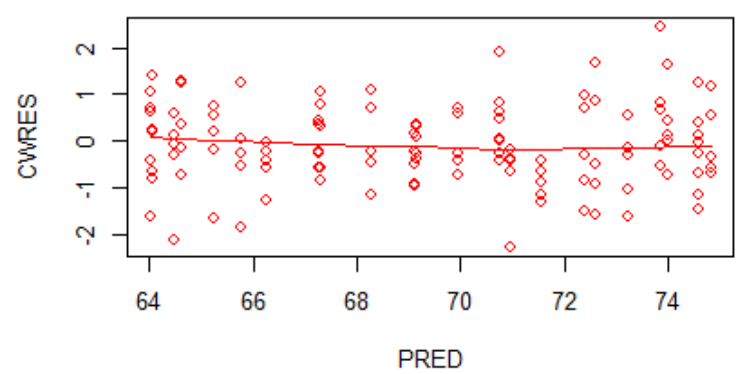

Figure 3. Conditional weighted residuals (CWRES) plotted against TIME (TOP) and typical predictions (PRED) (BOTTOM). Blue and red colors denote SBP and DBP, respectively (SBP, Systolic blood pressure; DBP, Diastolic blood pressure). 
suggesting that predicted values agree reasonably well with the observations. Figure 3 shows conditional weighted residuals plotted against time and predictions, demonstrating no appreciable trends.

Figures 4 and 5 show individual predictions superimposed on observations, indicating our model seems to offer sufficient flexibility to describe observed data.

For model validation, VPC in Figure 6 suggests that predictions are generally in agreement with observations in the 'dippers' where most of the median observed values fall within the

\section{Dippers}
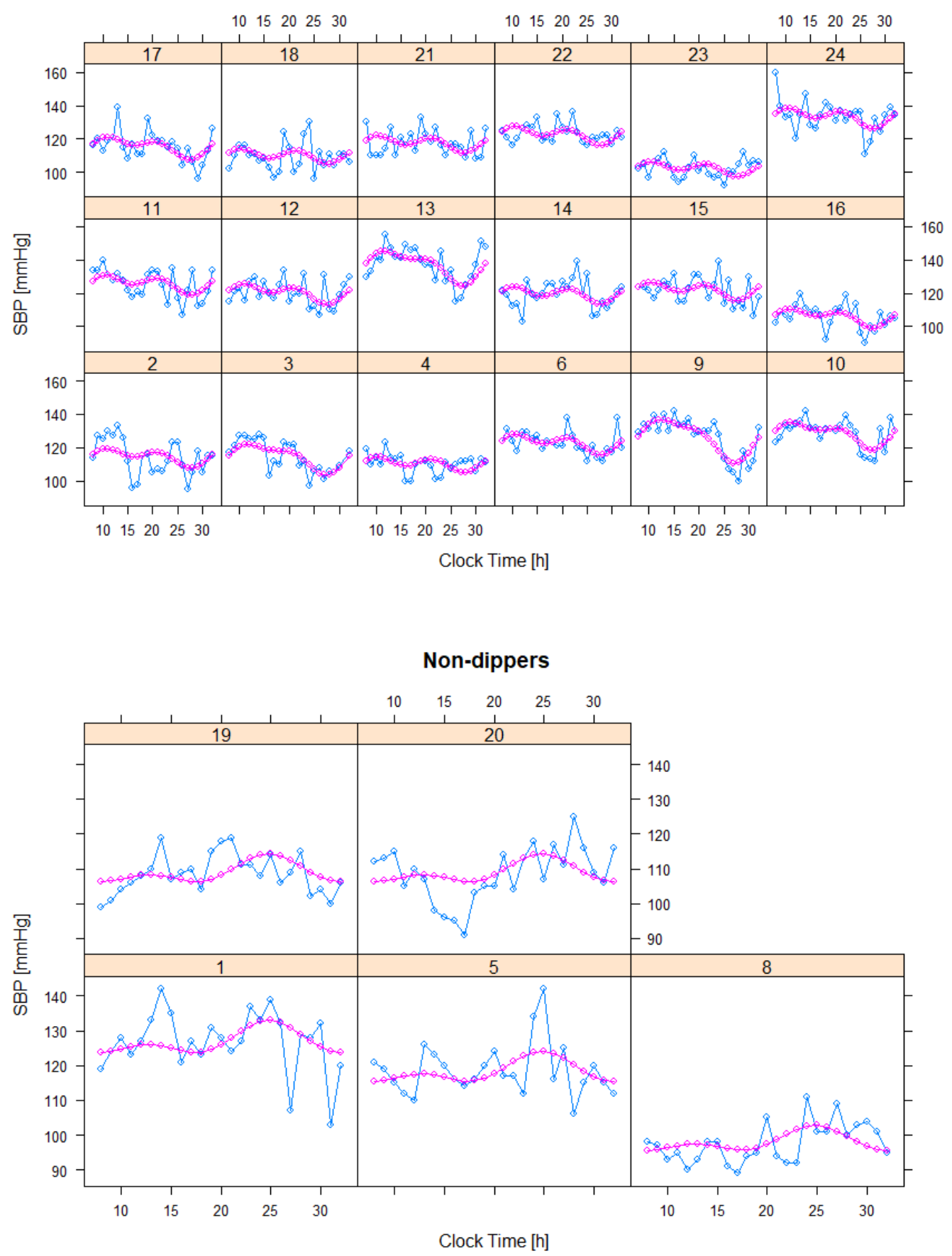

Figure 4. Individual predictions of SBP (red), superimposed on observations (blue), for dippers (TOP) and non-dippers (BOTTOM) (SBP, Systolic blood pressure). 
shaded areas depicting the $95 \%$ confidence intervals around the $5 \%, 50 \%$, and $95 \%$ percentiles. Concordance between the predicted and the observed values are not as good in the 'nondippers. Bootstrap was not carried out because the precisions of parameter estimates were reliable as judged by RSE, which was less than or about $30 \%$ in all cases, except for $46 \%$ of $A m p_{12}$ for DBP in the non-dipper group.

\section{Dippers}

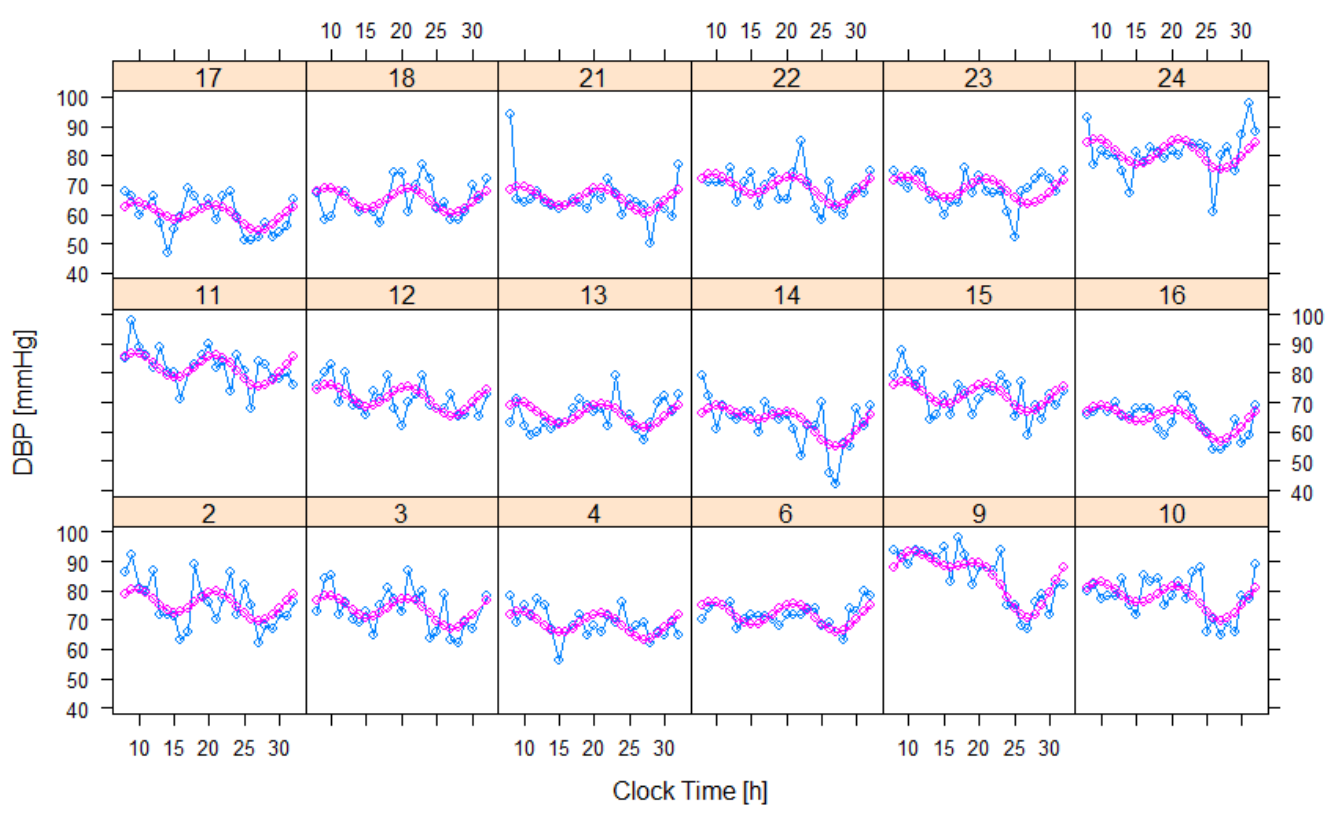

Non-dippers

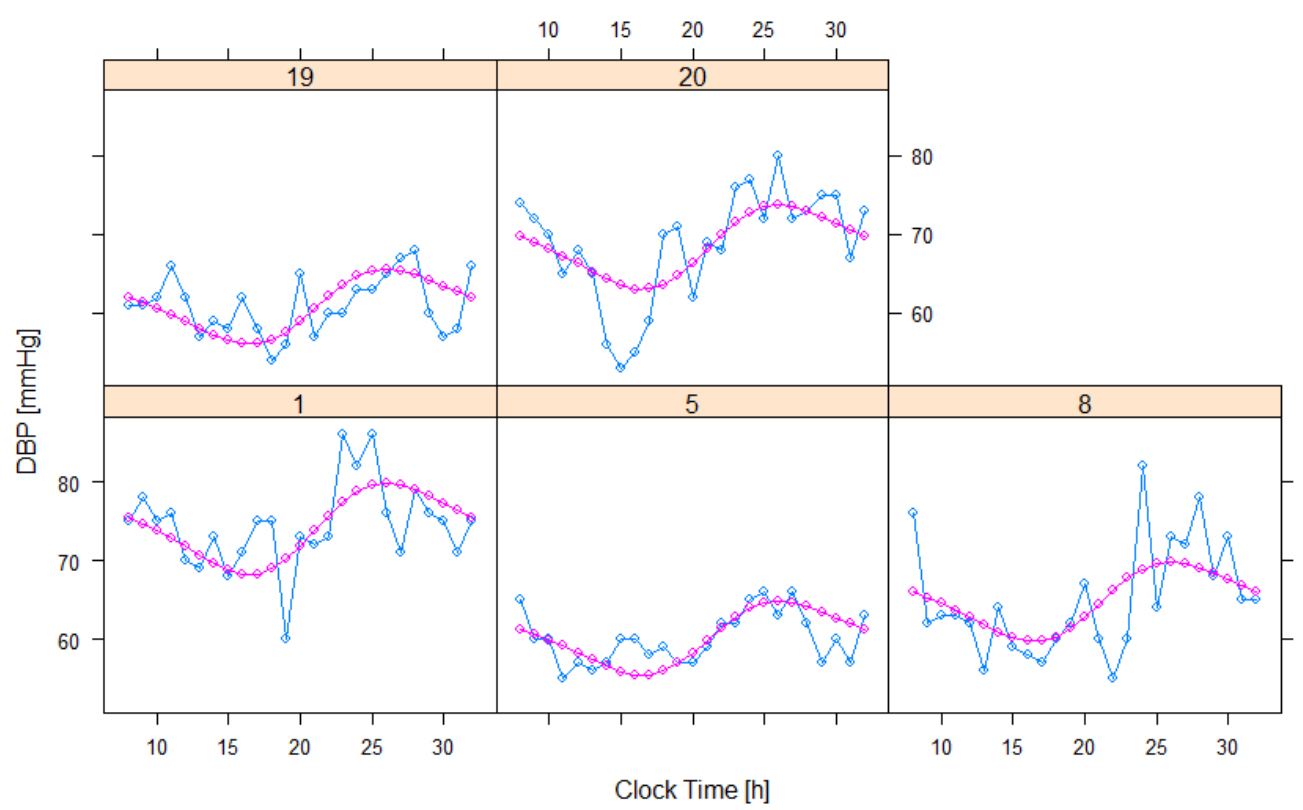

Figure 5. Individual predictions of DBP (red), superimposed on observations (blue), for dippers (TOP) and non-dippers (BOTTOM) (DBP, Diastolic blood pressure). 


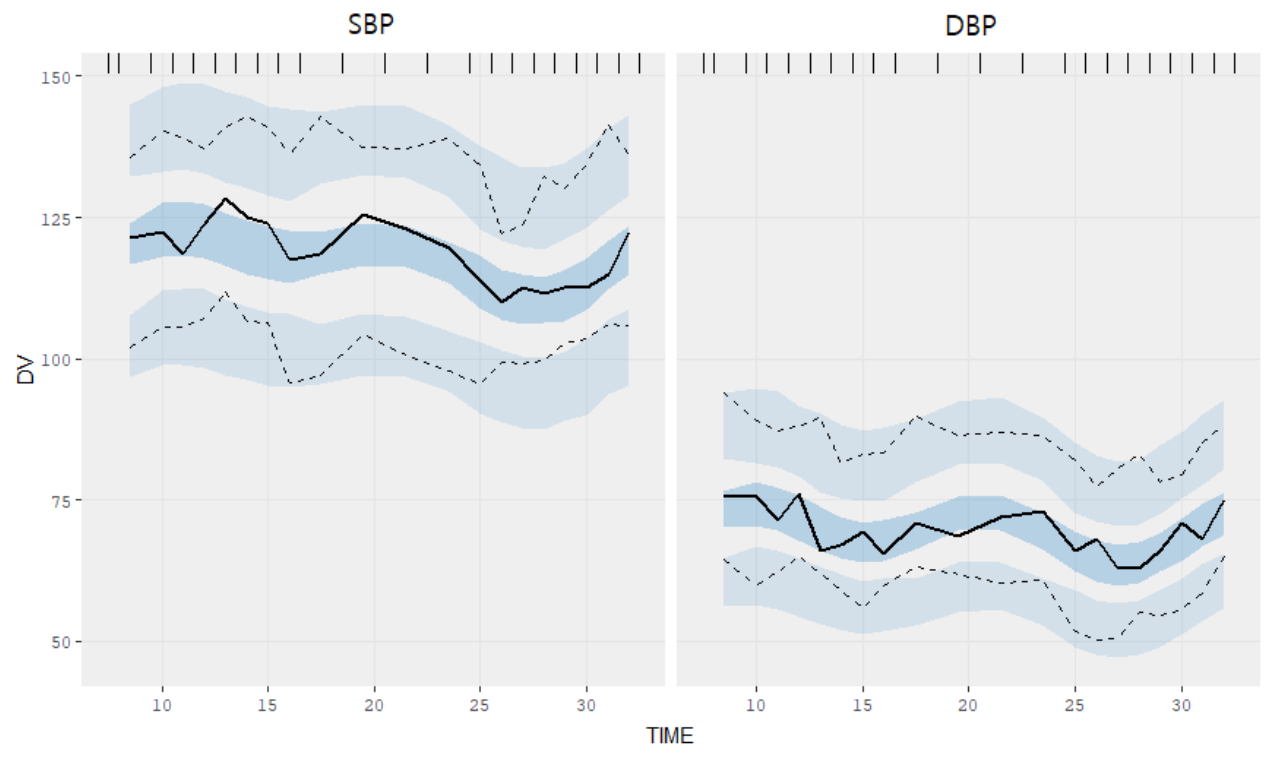

Subpopulation 2 (22\%)
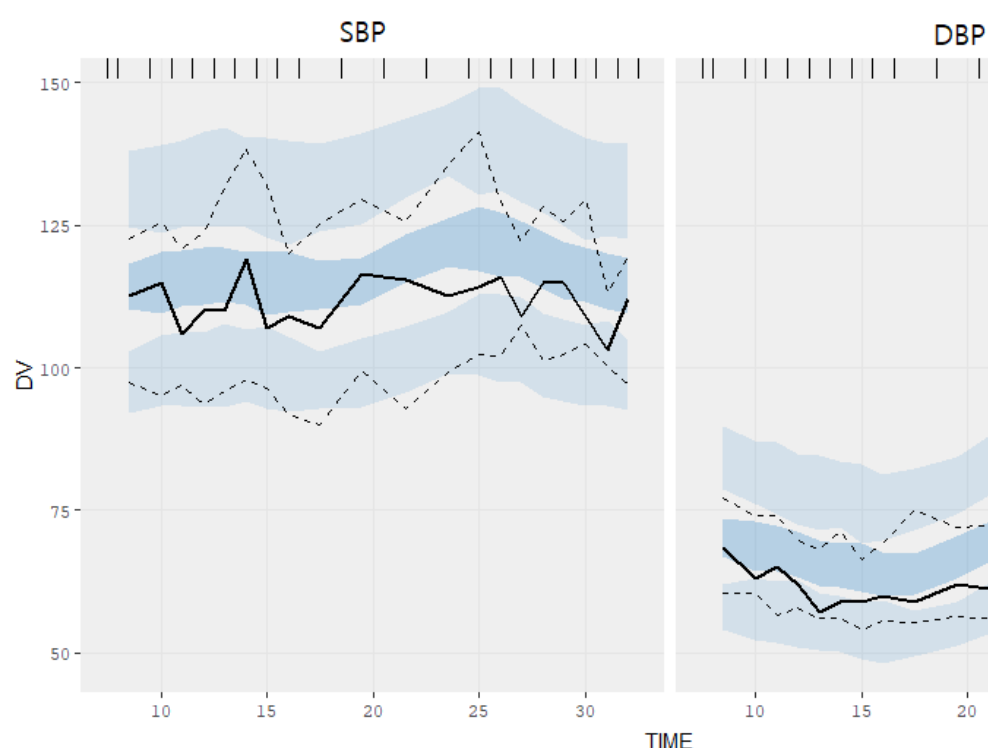

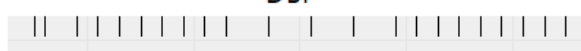

Figure 6. VPC of SBP (LEFT) and DBP (RIGHT) for dippers (TOP) and non-dippers (BOTTOM). The colored bands correspond to $95 \%$ confidence interval around $5 \%, 50 \%$, and $95 \%$ percentiles of the predictions, and the lines correspond to the median values of the corresponding percentiles of the observations (SBP, Systolic blood pressure; DBP, Diastolic blood pressure; DV, Dependent variable).

\section{Discussion}

The mixture model identified subgroups exhibiting different circadian blood pressure profiles. The model estimation results showed that about $80 \%$ of the study participants followed a typical pattern reported in the literature. The remaining $20 \%$ showed patterns that were more consistent with a non-dipper phenotype and exhibited differences in the acrophases (24.7 vs. $27.3 \mathrm{~h}$ ) associated with a 24 hours period oscillator. Such a phase difference inevitably leads to different circadian profiles between SBP and DBP (see Fig. 1) in non-dippers such that a tendency of rising SBP but falling DBP is seen in the morning. An empirical method of using a linear combination of cosine basis functions to model circadian profiles of blood pressure has been widely used, producing generally consistent and repro- 
ducible results. Staesson et al.[10] suggested using 4 harmonic oscillators (cosine functions) based on the Fourier model but in many papers, only 2 to 3 have been used. This cosine functions based approach to model circadian profiles is becoming one of the standard methods in pharmacometrics, $[8,11,12]$ and our work followed this approach. Of course, this is by no means the only method and an alternative approach of using a feedback turnover model that mimics asymmetric circadian oscillations has been reported.[13]

The non-dipper phenotype is associated with several disease states including stroke and cardiovascular complications of essential hypertension, and in an early Lancet paper,[3] 102 $(82.9 \%)$ out of 123 patients were classified as dippers while the remaining 21 (17.1\%) as non-dippers. These values are similar to the proportions estimated from our data of healthy subjects, indicating that the non-dipper subgroup showing atypical circadian profiles of blood pressure can exist in a population of healthy individuals.

Predicting circadian blood pressure profiles is important in the assessment of the antihypertensive drug effect. It is known that different antihypertensive agents show a maximal effect at different times. For example, oxprenolol taken during the day reduces daytime blood pressure but is less effective during the night and early morning.[14] Other drugs such as labetalol, nifedipine, and verapamil are known to show a more pronounced effect in the morning when the risk of cerebrovascular events is highest.[1]

Two of the major limitations of our work are the small sample size and failure to include female subjects. The latter limitation, however, would not pose a major problem when it comes to clinical trials for incrementally modified drugs since only male subjects are recruited in general. Inconsistency in the methods of BP measurement where study subjects were required to sit in the daytime but were allowed to be recumbent in the night could have led to another potential bias in the parameter estimates.

Notwithstanding these limitations, our work is the first to analyze baseline circadian BP profiles in the Korean populationbased non-linear mixed effects modeling methodology. The proportions of dippers and non-dippers and parameters related to the circadian profiles were successfully estimated. Our work is expected to serve as a useful reference in analyzing and interpreting data acquired from clinical trials assessing systematic intra-day blood pressure fluctuations and antihypertensive effects. It can also be applied to better assess drug safety of incrementally modified or generic drugs as measured by blood pressure change, which is currently in Korea assessed only based on the equivalence with the reference drug (so-called relative safety), not the placebo (so-called absolute safety).

\section{Acknowledgements}

This study was supported by the Brain Korea 21 Plus Project for Medical Science, Yonsei University

\section{Conflict of interest}

- Authors: The author declared no conflict of interest.

- Reviewers: Nothing to declare

- Editors: Nothing to declare

\section{References}

1. Sirgo MA, Mills RJ, DeQuattro V. Effects of antihypertensive agents on circadian blood pressure and heart rate patterns. Review. Arch Intern Med 1988;148:2547-2552.

2. Degaute JP, van de Borne P, Linkowski P, Van Cauter E. Quantitative analysis of the 24-hour blood pressure and heart rate patterns in young men. Hypertension 1991;18:199-210.

3. O'Brien E, Sheridan J, O'Malley K. Dippers and non-dippers. Lancet 1988;2:397.

4. Ohkubo T, Hozawa A, Yamaguchi J, Kikuya M, Ohmori K, Michimata M, et al. Prognostic significance of the nocturnal decline in blood pressure in individuals with and without high $24-\mathrm{h}$ blood pressure: the Ohasama study. J Hypertens 2002;20:2183-2189.

5. Metoki H, Ohkubo T, Kikuya M, Asayama K, Obara T, Hashimoto J, et al. Prognostic significance for stroke of a morning pressor surge and a nocturnal blood pressure decline: the Ohasama study. Hypertension 2006;47:149-154. doi: 10.1161/01.HYP.0000198541.12640.0f.

6. Che X, Mou S, Zhang W, Zhang M, Gu L, Yan Y, et al. The impact of nondipper circadian rhythm of blood pressure on left ventricular hypertrophy in patients with non-dialysis chronic kidney disease. Acta Cardiol 2017;72:149-155. doi: 10.1080/00015385.2017.1291133.

7. Baumgart P. Circadian rhythm of blood pressure: internal and external time triggers. Chronobiol Int 1991;8:444-450.

8. Tanigawara Y, Yoshihara K, Kuramoto K, Arakawa K. Comparative pharmacodynamics of olmesartan and azelnidipine in patients with hypertension: a population pharmacokinetic/pharmacodynamic analysis. Drug Metab Pharmacokinet 2009;24:376-388.

9. Chae D, Son M, Kim Y, Son H, Park K. Mechanistic model for blood pressure and heart rate changes produced by telmisartan in human beings. Basic Clin Pharmacol Toxicol 2018;122:139-148. doi: 10.1111/bcpt.12856.

10. Staessen J, Celis H, De Cort P, Fagard R, Thijs L, Amery A. Methods for describing the diurnal blood pressure curve. J Hypertens Suppl 1991;9: S16-S18.

11. Hempel G, Karlsson MO, de Alwis DP, Toublanc N, McNay J, Schaefer HG. Population pharmacokinetic-pharmacodynamic modeling of moxonidine using 24-hour ambulatory blood pressure measurements. Clin Pharmacol Ther 1998;64:622-635. doi: 10.1016/S0009-9236(98)90053-4.

12. van Rijn-Bikker PC, Snelder N, Ackaert O, van Hest RM, Ploeger BA, van Montfrans GA, et al. Nonlinear mixed effects modeling of the diurnal blood pressure profile in a multiracial population. Am J Hypertens 2013;26:11031113. doi: 10.1093/ajh/hpt088.

13. Sällström B, Visser SA, Forsberg T, Peletier LA, Ericson AC, Gabrielsson J. A pharmacodynamic turnover model capturing asymmetric circadian baselines of body temperature, heart rate and blood pressure in rats: challenges in terms of tolerance and animal-handling effects. J Pharmacokinet Pharmacodyn 2005;32:835-859. doi: 10.1007/s10928-005-0087-2.

14. Millar Craig MW, Mann S, Balasubramanian V, Raftery EB. Blood pressure circadian rhythm in essential hypertension. Clin Sci Mol Med Suppl 1978;4:391s-393s. 